\title{
Poor oral hygiene as trigger of diabetes mellitus progressiveness
}

\author{
Sunarko Setyawan \\ Physiology Department \\ School of Medicine Airlangga University \\ Surabaya - Indonesia
}

\begin{abstract}
Diabetes mellitus is a systemic disease with several major complications affecting both the quality and length of life. The disease is characterized by increasing susceptibility to infection that important risk factor for oral infection progressiveness; periodontitis, infection or lesions. Infection progressiveness and inflammation can increase blood cytokines. The cytokines modulate cells up and down regulation moreover apoptosis or necrosis cells. The increasing of the blood cytokines that implicate in the process of pancreatic $\beta$-cell destruction is not fully understood. Poor oral hygiene stimulate proinflammatory cytokines (such as: IL-1, IL6, TNF-alpha, etc.) and make chronic infection worse. IL-1 $\beta$ and/or TNF- $\alpha$ plus IFN- $\gamma$ induce $\beta$-cell apoptosis via the activation of $\beta$-cell gene networks under transcription controlling factors, such as NF- $\kappa \beta$ (nuclear factor- $\kappa \beta$ ) and STAT-1 (signal transducers and activators of transcription-1). Others mechanism of the decreased $\beta$-cell function may activate cytokines stimulated macrophages. The presence of activated macrophages within pancreatic islets in insulin-dependent diabetes mellitus suggests an involvement of $\beta$-cell death. This paper describes that poor oral hygiene are high predisposition on the diabetic progressiveness.
\end{abstract}

Key words: diabetes mellitus, cytokines, progressiveness

Correspondence: Sunarko Setyawan, c/o: Bagian Physiology, Fakultas Kedokteran Universitas Airlangga. Jln. Mayjend. Prof. Dr. Moestopo No. 47 Surabaya 60132, Indonesia.

\section{INTRODUCTION}

The oral cavity is a continuous source of infectious agents, and it often reflects progression of systemic pathologies or multiple disease. Diabetes is worldwide problem. The disease characterized by an increased susceptibility to infection, poor wound healing, and increased morbidity and mortality associated with disease progression. ${ }^{1-4}$ Diabetes is also recognized as an important risk factor for more severe and progressive periodontitis, infection or lesions resulting in the destruction of tissues and supporting bone that form the attachment around the tooth. ${ }^{1}$ The mechanistic studies have examined the potential effects of periodontal infection in the presence periodontal tissue destruction related to an altered inflammatory response. ${ }^{2,3,4}$ The connection between periodontal disease and diabetes based on information in the literature and to discuss proper management and referral of patients who have signs and symptoms of periodontal disease and other oral complications. Process of infection and inflammation can increase cytokines secretion in blood. Various oral complications of the booming cytokines on diabetes that implicated to the multiple organ diseases are unclear. ${ }^{3}$

Therefore the implication in the process of pancreatic $\beta$-cell destruction is not clearly understood. Proinflammatory secreted cytokines (IL-1, IL6, TNF-alpha, ect.) increase on poor oral hygiene. IL- $1 \beta$ and/or TNF- $\alpha$ plus IFN- $\gamma$ induce $\beta$-cell apoptosis via the activation of $\beta$-cell gene networks under the control of the transcription factors NF- $\kappa$ B and STAT-1.5,6 The others mechanism may be activated by macrophages, which its cytokine stimulated for necrotic process. The presence of activated macrophages within pancreatic islets in insulin-dependent diabetes mellitus suggests an involvement of $\beta$-cell death by necrosis of $\beta$-cell death. ${ }^{7}$ These investigations show which poor oral hygiene are high predisposition of diabetic progressiveness.

\section{Oral diabetic base problems}

Diabetes and periodontal disease are common chronic diseases observed in the U.S. population. These diseases are thought to be associated biologically, and a number of reviews and studies have proposed mechanisms to explain the relationship, including microvascular disease, changes in components of gingival crevicular fluid, changes in collagen metabolism, an altered host response, altered subgingival flora, genetic predisposition, and non enzymatic glycation. ${ }^{4}$

During infection in diabetes, inflammatory cytokines induce regulated changes in the host's internal milieu. Recent evidence indicates that these cytokines are constitutively produced, their production is increased by environmental stressors other than microbes, and they modulate "normal" physiological processes. ${ }^{8}$ Concentrations of pro-inflammatory cytokines are increased in the intestinal mucosa infection. Polymorphonuclear neutrophil granulocytes (PMN) are the most abundant cell type in intestinal lesions, but Interleukin 10 (IL-10) secretion is an important contra-inflammatory cytokine which induces down regulation of proinflammatory cytokines. ${ }^{9}$ 
Diabetes mellitus is a systemic disease with several major complications affecting both the quality and length of life. These oral complications are periodontal disease (periodontitis), decrease function of T cells, B cells and phagocytes on the oral cavity poor wound healing periodontal. ${ }^{10}$ The infection then leads to formation of pockets between the teeth and gums signaling breakdown of the periodontal apparatus and bone. ${ }^{11,12,13}$ In these factors may also be possible for the oral infection to predispose to systemic disease and this predisposes to chronic inflammation and periodontitis exacerbation. These processes can increases cytokines in blood. These cytokines can produce an insulin resistance syndrome similar to that observed in diabetes and initiate destruction of pancreatic beta cells leading to development of diabetes. It may also be possible for chronic periodontitis to induce diabetes. ${ }^{3,4}$

Diabetes can lead to disturbance of the salivary secretion that markedly increased dental caries, parotid gland enlargement, inflammation and fissuring of the lips (cheilitis), inflammation or ulcers of the tongue and buccal mucosa, oral candidiasis, salivary gland infection. ${ }^{14}$ Cracking, fissuring of the oral mucosa, burning mouth syndrome, and white lesions of the oral mucosa are increased in frequency. ${ }^{15}$ Chronic diabetes caused salivary hypo function. Salivary hypo function may predispose for secondary oral mucosal diseases. In these patients the protective coating of saliva is reduced or absent, leaving the oral mucosa more vulnerable can result in infection of the dental pulp and tooth abscess. ${ }^{11,13,15}$

\section{Systemic problem of inflammation}

Diabetes is chronic diseases and also recognized as an important risk factor for more severe and progressive infection and inflammation. ${ }^{1,15}$ E. coli administration significantly increased serum pro-inflammatory cytokines such as interleukin (IL)- $1 \beta$, IL-6, and tumor necrosis factor- $\chi$ and brain IL- $1 \beta$ levels beginning at the 6-h time point. ${ }^{16}$ Cytokines act in concert with specific cytokine inhibitors and soluble cytokine receptors to regulate the human immune response. Their physiologic role in inflammation and pathologic role in systemic inflammatory states are increasingly recognized. Major anti-inflammatory cytokines include interleukin (IL)-1 receptor antagonist, IL-4, IL-6, IL-10, IL-11, and IL-13 are recognized. ${ }^{17}$

Pro-inflammatory cytokines are IL-6, TNF- $\alpha$, IL- $1 \beta$, IL-8, IL-10 and IL-1 in the tissues inflammation likes rheumatoid arthritis that whom levels of IL-6, IL-8, TNF- $\alpha$, IL- $1 \beta$ and IL-10 were significantly elevated. ${ }^{18}$ Oral infections, especially periodontitis, as a causal factor for systemic diseases ${ }^{12}$. Infectious diseases and other sources of inflammation in lifetime exposure can decrease life quality of organs. In the cohorts across the life-span since 1751 in Sweden represents inflammatory processes that persist from early age into adult life. ${ }^{19}$

In vitro studies of human monocytes with diabetes have shown a hyper responsive phenotype with over expression of pro-inflammatory mediators such as interleukin- $1 \beta$
(IL-1 $\beta$ ), tumor necrosis factor- $\alpha$ (TNF- $\alpha$ ), and prostaglandin $\mathrm{E}_{2}$. In similar in vivo studies, patients with periodontitis and diabetes were found to have significantly higher levels of local inflammatory mediators compared to systemically healthy individuals with periodontal disease. ${ }^{20}$ IL-1 may exert neurotoxic or neuroprotective actions. Interleukin-1 (IL-1) participates in diverse forms of brain damage including ischemia, brain trauma, and excitotoxic injury. Administration of low doses of IL-1 markedly exacerbates these forms of brain damage, whereas blocking IL-1 release or actions reduces neuronal death. IL-1 receptor antagonist (IL-1ra) is also up regulated by brain damage (mainly by neurons) and acts as an endogenous inhibitor of neuron degeneration, presumably by blocking IL-1 actions on its receptor that can damage to pancreas cells. ${ }^{5,21}$

Concentrations of pro-inflammatory cytokines are increased in the intestinal mucosa infection. Polymorphonuclear neutrophil granulocytes (PMN) are the most abundant cell type in intestinal lesions, but interleukin 10 (IL-10) secretion is an important contra-inflammatory cytokine which induces down regulation of proinflammatory cytokines and plays a protective role in blood vessels during diabetes. ${ }^{8,22,23}$ IL-10 is an immunosuppressive cytokine or induces down regulation of proinflammatory cytokines in the immune system. IL-19 belongs to the IL-10 family, which includes IL-10, IL-19, IL-20, IL-22. Little is known about the biologic function and gene regulation of IL-19. To understand the gene regulation of human IL-19, we identified a human IL-19 genomic clone and analyzed its promoter region. Treatment of monocytes with mouse IL-19 induced the production of IL-6 and TNF- $\alpha$. It also induced mouse monocyte apoptosis and the production of reactive oxygen species. The results indicated that mouse IL-19 may have some important roles in inflammatory responses due to the regulation of IL- 6 and TNF- $\alpha$ and induces apoptosis. ${ }^{8,24}$

\section{DISCUSSION}

\section{Modulation cytokine in pancreas damage}

Pancreatic Beta cells damages are caused by mechanism of (1) apoptotic (2) acidosis were increased inflammatory and necrotic. Tissue acidosis is an important feature of inflammation. Acid-sensing ion channels (ASICs) transcript levels were increased in inflammatory conditions in vivo. Now, we have found that this increase is caused by the proinflammatory mediators NGF, serotonin, interleukin1 , and bradykinin. IL- 1 is a proinflammatory cytokine that plays important roles in inflammation. However, the role of this cytokine under physiological conditions is not known completely. These observations suggest that IL-1 plays an important role in lipid metabolism by regulating insulin levels and lipase activity under physiological conditions. ${ }^{25}$ Interleukin-1ß appears to be among the most important inflammatory mediators, causing pancreatic islet dysfunction and apoptosis through the up-regulation of 
inducible nitric oxide (NO) synthase. ${ }^{26}$ Cytokines have been shown to have dramatic effects on pancreatic islets and insulin-secreting beta-cell lines. It is well established that cytokines such as interleukin-1beta (IL-1beta), tumor necrosis factor-alpha (TNF-alpha), and gamma-interferon (IFN-gamma) inhibit beta-cell function and are cytotoxic to human and rodent pancreatic islets in vitro. ${ }^{5}$ The indicate that IL-1 beta-stimulated JNK (c-Jun $\mathrm{NH}_{2}$-terminal kinase) activity may be distinctly targeted to cytoplasmic and/or membrane compartments in clonal insulin-producing cells, and that JIP (JNK-interacting protein) may serve to localize JNK activity to specific substrates. ${ }^{27}$ Decreasing of intracellular JNK signaling and confer long-term protection to pancreatic beta-cells from IL-1beta-induced apoptosis

Type 2 diabetes. Advances in the molecular biology of insulin resistance and $\beta$-cell dysfunction increasingly support a role for inflammatory mediators, particularly cytokines, and elements of the innate immune system in the pathogenesis of type 2 diabetes. Cytokine production as a consequence of an infectious challenge could potentially contribute to insulin resistance in a number of ways, including 1) modification of insulin receptor substrate- 1 by serine phosphorylation, 2) alteration of adipocyte function with increased production of free fatty acids, and 3) diminution of endothelial nitric oxide production. ${ }^{28,29}$ In fact, cytokine-induced mechanisms have been suggested to participate in the $\beta$-cell damage.

Type 1 diabetes. Type 1 diabetes is an organ-specific autoimmune disease characterized by the destruction of the B-cells within the islets of Langerhans. The elimination of B-cells is caused by self-reactive T-cells that infiltrate the pancreatic islets (insulitis). Controversy exists as to the events that initiate the activation of the islet-reactive Tcells. However, many studies have established that apoptosis is the major mechanism by which $\beta$-cells are destroyed. A possible role for macrophage defective phagocytosis in the pathogenesis of type 1 diabetes. The ß-cell autoimmune destructions are induced by interventions that block B-cell destructive leukocytes ( $\mathrm{T}$ cells and macrophages) and their cytotoxic products (e.g., cytokines, perforin, granzymes, FasL/Fas). Type 1 diabetes is an organ-specific autoimmune disease that is mediated by autoreactive $\mathrm{T}$ cells. It showed that administration of a soluble dimeric peptide "major histocompatibility complex (pMHC) class II chimera (DEF). Autoreactive CD4+ $\mathrm{T}$ cells via alteration of early $\mathrm{T}$ cell receptor signaling and stimulation of interleukin 10 "secreting T regulatory type 1 cells in the pancreas. Soluble dimeric pMHC class II may be useful in the development of immunospecific therapies for type 1 diabetes. Analysis of the autoimmune nature of type 1 diabetes has led to attempts at prevention by two major approaches. First, the anti-inflammatory cytokines interleukin 4 (IL-4), IL-10, IL-11, IL-13 and transforming growth factor- $\beta$ (TGF- $\beta$ ), antibodies specific for the proinflammatory cytokines interferon- $\gamma($ IFN- $\gamma)$ and tumor necrosis factor- $\alpha$ (TNF- $\alpha$ ), TCR-CD3 complex, CD4 and CD8 coreceptors, CD25 activation marker or CD40 ligand
(CD40L) and B7-2 costimulatory molecules. These reagents have a variety of side effects that call into question the ethics of applying them to asymptomatic humans. Second, there is the possibility of using immunospecific therapy to induce tolerance in autoreactive T cells. ${ }^{30}$

Macrophage interactions with apoptotic cells can suppress inflammatory responses, but cell death by apoptosis may triggered by inflammation. TLR ligands induced early and sustained secretion of TNF- $\alpha$, macrophage-inflammatory protein (MIP) $1 \alpha$ and MIP-2 with later secretion of IL-10, IL-12, and TGF- $\beta 1$; apoptotic cells alone stimulated late TGF- $\beta 1$ secretion only. The combination of apoptotic cells and TLR ligands enhanced early secretion of TNF- $\alpha$, MIP- $1 \alpha$, and MIP- 2 and increased late TGF- $\beta 1$ secretion, while suppressing late TNF- $\beta$, IL-10, and Il-12 by mechanisms which could nevertheless be overridden by IFN- $\gamma \cdot{ }^{31}$ In addition to their well known immune and proinflammatory activities, IFNs possess homeostatic functions that limit inflammation and tissue destruction in a variety of conditions. The mechanisms underlying the homeostatic actions of IFNs are not well understood. IFNs completely suppressed the activation of IL-1 signal transduction pathways in macrophages. The mechanism of IFN-mediated inhibition of IL-1 action and signaling was modulation of IL-1R expression, which was also observed in vivo. IFN- $\gamma$-mediated down-regulation of IL-1R type I expression was dependent on Stat1, a transcription factor typically considered to be a key mediator of macrophage activation by IFNs. These results identify cellular and molecular mechanisms that contribute to the homeostatic role of IFNs in limiting inflammation and associated tissue destruction. ${ }^{32}$ Other addition to apoptosis being the main mechanism by which $\beta$ cells are destroyed, $\beta$-cell apoptosis has been implicated in the initiation of type 1 diabetes mellitus through antigen crosspresentation mechanisms that lead to $\beta$-cell-specific T-cell activation. Caspase- 3 is the major effector caspase involved in apoptotic pathways. Despite evidence supporting the importance of $\beta$-cell apoptosis in the pathogenesis of type 1 diabetes, the specific role of caspase- 3 in this process is unknown. ${ }^{33}$ Fas seems to be implicated in $\beta$-cell apoptosis via an intracellular death domain and proinflammatory cytokines can induce up-regulation of Fas expression on $\beta$-cells, making them susceptible to apoptosis in the presence of agonistic anti-Fas antibodies or interaction with Fas-ligand (FasL, CD95L)-expressing T-cells. The role of Fas in $\beta$-cell apoptosis is still under debate and has been challenged by several studies. In addition, up-regulation of several anti-apoptotic members of the Bcl-2 family of proteins, such as Bcl-2 and Bcl-xL has been closely associated with increased resistance to apoptosis and potentially to diabetes susceptibility. ${ }^{34}$

\section{Diabetic progressiveness on the quality of oral hygiene}

Periodontitis can cause factor for systemic diseases. ${ }^{12}$ Infectious diseases and other sources of inflammation in oral are excessively, especially adult life. ${ }^{19}$ Uncontrolled 

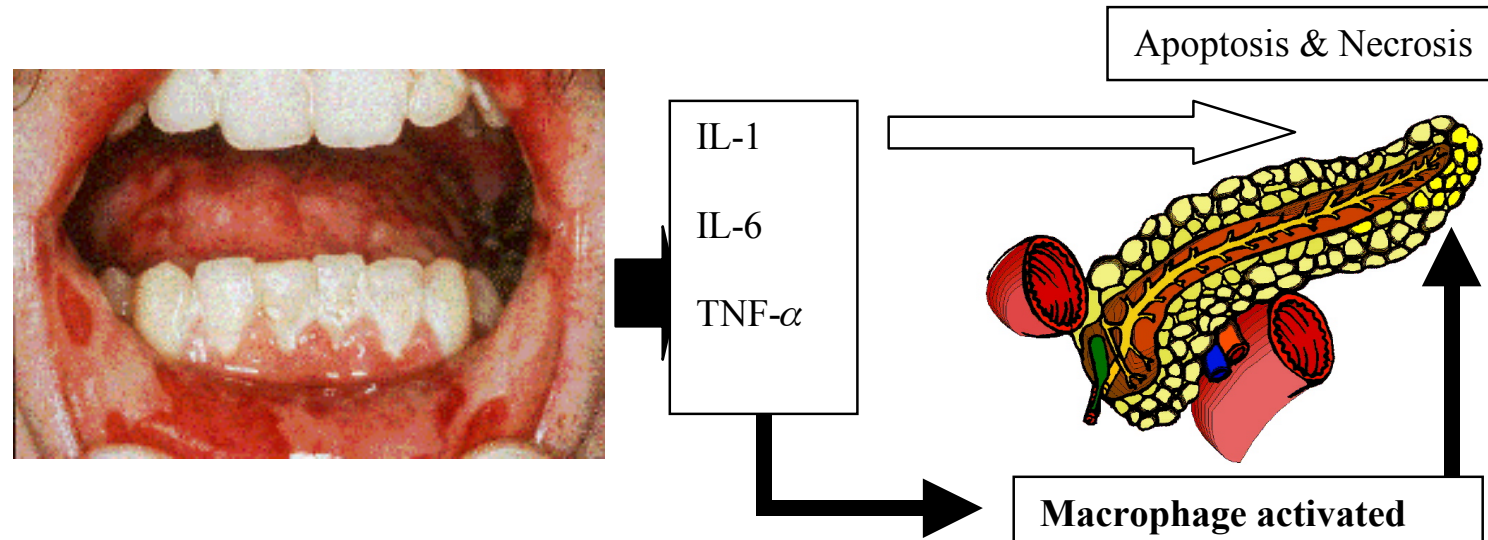

Apoptosis \& Necrosis

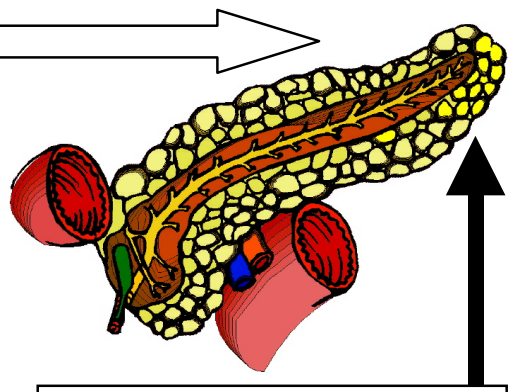

Macrophage activated

Figure 1. Mechanisms of $\beta$-cell apoptosis and necrosis.

diabetes and low of oral hygiene evoked chronic infection and diabetic progressiveness. ${ }^{3,4,11}$ Infection progressiveness and inflammation can be fluctuation of blood cytokines. 8,20 The blow up blood cytokines that implicated in the process of pancreatic $\beta$-cell destruction is not fully understood. Secretion of proinflammatory cytokines (IL-1, IL6, TNFalpha, ect) increase on poor oral hygiene (infection and inflammation). IL- $1 \beta$ and/or TNF- $\alpha$ plus IFN- $\beta$ induce $\beta$-cell apoptosis via the activation of $\beta$-cell gene networks under the control of the transcription factors NF- $\kappa \mathrm{B}$ and STAT-1.5,24,26,32,35 This argues against a unifying hypothesis for the mechanisms of $\beta$-cell death in type 1 and type 2 diabetes and suggests that different approaches will be required to prevent $\beta$-cell death in type 1 and type 2 diabetes. ${ }^{36}$ Others investigation showed that combination of IL- $1 \beta$ plus interferon- $\gamma$ causes a time-dependent increase in apoptotic cells in the $\beta$-cells (Figure 1). ${ }^{6}$

The others mechanism of the decreased $\beta$-cell function maybe activated macrophages. The presence of activated macrophages within pancreatic islets in insulin-dependent diabetes mellitus suggests an involvement of B-cell death by necrosis of $ß$-cell death. ${ }^{37}$ These investigations show that poor oral hygiene are high predisposition of diabetic progressiveness.

Diabetes is chronic diseases and also recognized as an important risk factor for more severe and progressive infection and inflammation in oral. Infection progressiveness and inflammation in oral can increase blood proinflammatory cytokines. Incremental of the cytokines may play important roles in pancreatic B-cells islet destruction. Diabetic Patient ought to preserve oral hygiene to avoid progressiveness these disease.

\section{REFERENCES}

1. Wild S, Roglic G, Green A, Sicree R, King H. Global prevalence of diabetes: estimates for the year 2000 and projections for 2030 . Diabetes Care 2004;27:1047-53.

2. Tsai C, Hayes C, Taylor GW. Glycemic control of type 2 diabetes and severe periodontal disease in the US adult population. Community Dent Oral Epidemiol 2002;30:182-92.
3. Southerland JH, Taylor GW, Offenbacher S. Diabetes and periodontal infection: making the connection. Clinical Diabetes 2005; 23:171-8.

4. Lacopino AM Periodontitis and diabetes interrelationships: role of inflammation. Ann Periodontol 2001; 6:125-37.

5. Hoorens A, Stangé G, Pavlovic D, Pipeleers D. Distinction between Interleukin-1-induced necrosis and apoptosis of islet cells. Diabetes 2001; 50:551-7.

6. Van de Casteele M, Kefas BA, Ling Z, Heimberg H, Pipeleers DG. Specific expression of $\mathrm{Bax}-\omega \mathrm{n}$ pancreatic $\beta$-cells is down-regulated by cytokines before the onset of apoptosis. Endocrinology 2002; 143(1):320-6.

7. Saldeen J. Cytokines induce both necrosis and apoptosis via a common Bcl-2-inhibitable pathway in Rat insulin-producing cells. Endocrinology 2000; 141(6):2003-10.

8. Cannon JG. Inflammatory cytokines in no pathological states. News in Physiological Sciences 2000; 15(6):298-303.

9. Nikolaus S, Bauditz J, Gionchetti P, Witt C, Lochs H, Schreiber S. Increased secretion of pro-inflammatory cytokines by circulating polymorph nuclear neutrophils and regulation by interleukin 10 during intestinal inflammation. GUT 1998; 42:470-6.

10. Giannobile WV. C-telopeptide pyridinoline cross-links: Sensitive indicators of periodontal tissue destruction. Annals of the New York Academy of Sciences 1999; 878:404-12.

11. Atkinson JC, O'Connell A, Aframian D. Oral manifestations of primary immunological diseases. Am Dent Assoc 2000; 131(3): 345-56.

12. Taylor GW, Burt BA, Becker MP, Genco RJ, Shlossman M. Glycemic control and alveolar bone loss progression in type 2 diabetes. Ann Periodontol 1 1998; 3:30-39.

13. Li X, Kolltveit KM, Tronstad L, Ingar Olsen I. Systemic diseases caused by oral infection. Clinical Microbiology Reviews 2000; 13(4):547-58.

14. Bauroth K, Charles CH, Mankodi SM, Simmons K, Zhao Q, Kumar LD. The efficacy of an essential oil antiseptic mouth rinse vs. dental floss in controlling interproximal gingivitis: A comparative study. J Am Dent Assoc 2003; 134:359-65.

15. Geerlings SE, Hoepelman AI. Immune dysfunction in patients with diabetes mellitus (DM). FEMS Immunol Med Microbiol 1999; 26:259-65.

16. Jensena JL, Barkvoll P. Clinical implications of the dry mouth: Oral mucosal diseases. Annals of the New York Academy of Sciences 1998; 842:156-62.

17. Campisi JMK, Hansen MK, O’Connor KA, Biedenkapp JC, Watkins LR, Maier SF, Fleshner M. Circulating cytokines and endotoxin are not necessary for the activation of the sickness or corticosterone response produced by peripheral E. coli challenge. J Appl Physiol 2003; 95:1873-82.

18. Opal SM, DePalo VA. Anti-inflammatory cytokines. Chest 2000; 117:1162-72. 
19. Nowlan ML, Drewe E, Bulsara H, Esposito N, Robins RA, Tighe PJ, Powell RJ, Todd I. Systemic cytokine levels and the effects of etanercept in TNF receptor-associated periodic syndrome (TRAPS) involving a C33Y mutation in TNFRSF1A. Rheumatology 2006; 45(1):31-37.

20. Finch CE, Crimmins EM. Inflammatory exposure and historical changes in human life-spans. Science 2004; 305(5691):1736-9.

21. Salvi GE, Beck JD, Offenbacher S. PGE2, IL-1 beta, and TNF-alpha responses in diabetics as modifiers of periodontal disease expression. Ann Periodontol 1998; 3:40-50.

22. Stuart M. The role of pro- and antiinflammatory cytokines in neurodegeneration. Annals of the New York Academy of Sciences 2000; 917:84-93.

23. Gunnett GA, Heistad DD, Berg DJ, Faraci FM. IL-10 deficiency increases super oxide and endothelial dysfunction during inflammation. Am J Physiol Heart Circ Physiol 2000; 279:1555-1562

24. Gunnett CA, Heistad DD, Faraci FM. Interleukin-10 protects Nitric Oxide-dependent relaxation during diabetes. Role of super oxide. Diabetes 2002; 51:1931-7.

25. Liao YC, Liang WG, Chen FW, Hsu JH, Yang JJ, Chang MS. IL-19 induces production of IL- 6 and TNF- $\alpha$ and results in cell apoptosis through TNF- $\alpha$. The Journal of Immunology 2002; 169:4288-97.

26. Matsuki T, Horai R, Sudo K, Iwakura Y. IL-1 plays an important role in lipid metabolism by regulating insulin levels under physiological conditions. The Journal of Experimental Medicine 2002; 198(6):877-88.

27. Moulin N, Widmann C. Islet-Brain (IB)/JNK-Interacting proteins (JIPs): Future targets for the treatment of neurodegenerative diseases?. Current Neurovascular Research 2004; 1(2):111-27.

28. Barshes NR, Wyllie S, Goss JA. Inflammation-mediated dysfunction and apoptosis in pancreatic islet transplantation: implications for intrahepatic grafts. Journal of Leukocyte Biology 2005; 77:587-97.
29. Hotamisligi GS, Peraldi P, Budavari A, Ellis R, White MF, Spiegelman BM. IRS-1-mediated inhibition of insulin receptor tyrosine kinase activity in TNF-alpha- and obesity-induced insulin resistance. Science 1996; 271:665-8.

30. Kolb H, Poulsen MT. An immune origin of type 2 diabetes? Diabetologia 2005; 48:1038-50.

31. Casares S, Hurtado A, McEvoy RC, Sarukhan A, von Boehmer H, Brumeanu TD. Down-regulation of diabetogenic $\mathrm{CD} 4{ }^{+} \mathrm{T}$ cells by a soluble dimeric peptide - MHC. 2002.

32. Lucas M, Stuart LM, Savill J, Hulbert LA. Apoptotic cells and innate immune stimuli combine to regulate macrophage cytokine secretion. The Journal of Immunology 2003; 171:2610-5.

33. Hu X, Ho HH, Lou O, Hidak C, Ivashkiv LB. Homeostatic role of interferons conferred by inhibition of IL-1-mediated inflammation and tissue destruction. The Journal of Immunology 2005; 175:131-8.

34. Liadis N, Murakami K, Eweida M, Elford AR, Sheu L, Gaisano HY, Hakem R, Ohashi PS, Woo M. Caspase-3-dependent B-cell apoptosis in the initiation of autoimmune diabetes mellitus. Molecular and Cellular Biology 2005; 25(9):3620-9.

35. Gil MJM, Diosdado AM. Assay for high glucose-mediated islet cell sensitization to apoptosis induced by streptozotocin and cytokines. Biol Proced Online 2005; 7:162-71.

36. Ohara-Imaizumi M, Cardozo AK, Kikuta T, Eizirik DL, Nagamatsu $\mathrm{S}$. The cytokine interleukin- $1 \beta$ Reduces the docking and fusion of insulin granules in pancreatic $\beta$-cells, preferentially decreasing the first phase of exocytosis. J Biol Chem 2004; 279(40):41271-4.

37. Cnop M, Welsh N, Jonas JC, Jörns A, Lenzen S, Eizirik DL. Mechanisms of pancreatic B-cell death in type 1 and type 2 diabetes. Diabetes 2005; 54:S97-S107. 